\title{
Modelo experimental con bloques aleatorios simples y análisis multivariado para el mejoramiento de procesos orgánicos en la agroindustria
}

\section{Mauricio Bustamante Jamid*}

Sandra Valbuena Antolinez

Experimental Model with Simple

Random Blocks and Multivariate

Analysis for the Improvement of

Drganic Processes in Agroindustry

Madèle expérimental de blacs aléatoires simples et analyse multivariée pour l'améliaration des processus organiques dans l'industrie agroalimentaire

Madelo experimental cam blocus aleatórios simples $\mathrm{e}$ análise multivariado para $\square$ melhoramento de processos orgânicus na agruindústria

"Maestría en Ingeniería Industrial, Universidad Distrital Francisco Fosé de Caldas; Ingeniería Comercial con énfasis en modelos econométricos, Universidad de Ciencias Aplicadas y Ambientales (UDCA) 


\section{RESUMEN}

En Colombia, el estudio y diseño experimental enfocado en procesos agroindustriales, es limitado. Es evidente cómo se han desaprovechado las ventajas comparativas que posee el País, haciendo referencia a las características climáticas, al igual que las ventajas que pueden ofrecer diversas especies, que obedeciendo a su situación geográfica, beneficiarían un desarrollo tanto social como comercial, así como el uso de productos orgánicos, soportado en procesos de investigación que hagan uso de modelos experimentales para la optimización de los procesos. De tal manera que las pocas alternativas establecidas, sumadas a la carencia de infraestructura adecuada, impiden avances a gran escala en este sector económico, entorpeciendo las condiciones de competitividad necesarias en el contexto global (Faramillo, 1990-2000).

\section{ABSTRACT}

In Colombia, the study and experimental design focusing on agro industrial processes is limited. It is evident that comparative advantages of the country have been misused regarding climatic conditions, as well as the advantages that our species offer, which being favored by our geographical situation would benefit social and commercial development and the use of organic products, supported by research processes that apply experimental models to optimize operations. Consequently, few stablished alternatives together with an appropriate infrastructure affect the economic sector at a high scale, avoiding the necessary conditions for competitiveness, which are fundamental in a global context (Faramillo C. F., 1990-2000).

\section{Malabras clave}

Diseño experimental Agroindustria Agricultura limpia Análisis de varianza Tratamientos experimentales Bloques aleatorios simples
Key words

Experimental design Agroindustry

Clean agriculture

Analysis of variance Experimental treatments Simple random blocks Competitiveness 


\section{RESUMÉÉ}

La recherche et les analyses expérimentales axées sur les procédés agro-industriels étant limitées en Colombie, les avantages comparatifs que possède ce pays n'ont malheureusement pas été mis à profit. Les caractéristiques climatiques dues à sa position géographique tout comme les avantages que peuvent offrir les nombreuses espèces bénéficieraient au développement social et commercial de la population. L'utilisation de produits organiques combinée à des processus de recherche utilisant des modèles expérimentaux pour l'optimisation des processus n'offrent que peu d'alternatives car le manque d'infrastructures adéquates pose une entrave aux conditions de compétitivité dans un contexte mondial (Faramillo C. F., 1990-2000).

\section{RESUMO}

$\mathrm{Na}$ Colômbia o estudo e desenho experimental enfocado nos processos agroindustriais é limitado, é evidente como se desperdiçam as vantagens comparativas que possui o país, fazendo referência às características climáticas, igualmente as vantagens que podem oferecer diversas espécies, que obedecendo a sua situação geográfica beneficiariam um desenvolvimento tanto social como comercial, assim como o uso de produtos orgânicos, documentados em processos de pesquisa científica que façam uso de modelos experimentais para a otimização dos processos; de tal maneira que as poucas alternativas estabelecidas, somadas a carência de infraestrutura adequada impedem avanços a grande escala no setor econômico, dificultando as condições de competitividade, necessárias no contexto global (Faramillo C. F., 1990-2000).

\section{Mots clefs}

Industrie agro-alimentaire Agriculture propre Analyse de la variance Traitements expérimentaux Blocs aléatoires simples

Compétitivité

\section{Palavras-chave}

Desenho experimental Agroindústria Agricultura limpa Análise de variantes

Tratamentos experimentais Simple random blocks Competitividade 


\section{INTRODUCCIÓN}

$\mathrm{E}$ n Colombia, el estudio y diseño experimental enfocado en procesos agroindustriales es limitado. Es evidente cómo se han desaprovechado las ventajas comparativas que posee el país, haciendo referencia a las características climáticas, al igual que las ventajas que pueden ofrecer diversas especies, que obedeciendo a su situación geográfica beneficiarían un desarrollo tanto social como comercial, así como el uso de productos orgánicos, soportado en procesos de investigación que hagan uso de modelos experimentales para la optimización de los procesos; de tal manera que las pocas alternativas establecidas, sumadas a la carencia de infraestructura adecuada impiden avances a gran escala en este sector económico, entorpeciendo las condiciones de competitividad, necesarias en el contexto global (Jaramillo C. F., 1990-2000).

Los indicadores presentados por el Departamento de Planeación Nacional (DPN, 2014) para el sector agropecuario, se enfocan en la superficie cosechada, el rendimiento de la cosecha y la

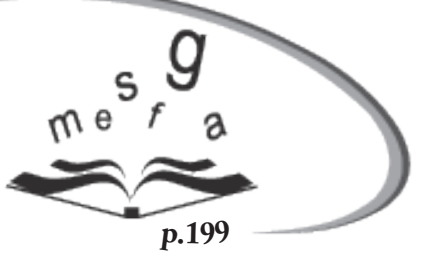

producción total; así mismo, los programas de misión para la transformación para el campo colombiano (DPN D. d., 2015) se centran en el cierre de las brechas sociales, desde la dotación de condiciones de interés social, la inclusión productiva de los pequeños productores, el desarrollo de la ruralidad competitiva a partir de la estabilidad macroeconómica, además de un soporte por medio de los bienes públicos, dónde se incluye tecnología, infraestructura de transporte, energía y TIC; adecuación de tierras, protección de los derechos de propiedad; información sobre mercados, seguridad y justicia, el desarrollo ambiental sostenible y una reforma institucional profunda.

Todo lo anterior, se considera pertinente para generar unas condiciones mínimas de estabilidad para el sector, sin embargo, si se hace prospección para el desarrollo, se requiere de la investigación que compruebe el uso de las prácticas de tal forma que permita el mejoramiento de los procesos y la inclusión de lo orgánico como una estrategia de sostenibilidad, por lo tanto, se presenta un caso de aplicación de experiemntación.

En la propuesta de Colciencias, desde las ciencias, tecnología e innovación agropecuarias, se resaltan las siguientes líneas de actuación que tienen una relación con la experimentación y factibilidad de aplicación (Colciencias, 2012):

- Uso de biotecnología para caracterización de materiales y rasgos genéticos de interés estratégico para mejoramiento genético (mayor adaptabilidad, resistencia a plagas y enfermedades).

- Producción de bioinsumos (bioplaguicidas, biofertilizantes).

- Necesidad y oportunidad de dar mayor valor agregado (café, frutas y hortalizas, pescados, caña de azúcar, caña panelera, cacao, papa).

- Mejoramiento de procesos de transformación y aumento de la capacidad productiva

En el mundo, la tendencia hacia la utilización de productos orgánicos está en crecimiento. En 2002, las ventas de estos productos alcanzaron 23 mil millones de dólares, superando los 19 mil millones de dólares alcanzados en 2001. El mercado de los Estados Unidos registra el primer lugar en ventas de productos orgánicos con un valor de 11.75 mil millones de dólares en 2002; el mercado alemán ocupa el segundo lugar, con 3.06 mil millones de dólares; y el mercado británico el tercer lugar, con un valor de 1.5 mil millones de dólares (Gómez Tovar \& Cruz, 2004). Por lo tanto, sí Colombia se quiere considerar un país agrícola, debe invertir esfuerzos en investigación y en el desarrollo 
experimental para la generación de alternativas y diversificación de los procesos agrícolas; una opción son los productos orgánicos que le permita desarrollar el mercado doméstico e internacional.

El desarrollo científico en agricultura limpia es mínimo, situación que trae como consecuencia la limitación de la participación nacional en mercados ecológicos, puesto que la calidad de los productos es escasa debido en primer lugar, al mantenimiento de procesos transgénicos y químicos como elección permanente en el manejo agrícola; en segundo lugar, los análisis se desarrollan en algunos sectores únicamente de forma nominal, conservando en la práctica un carácter empírico, de tal forma que en el transcurso de los procesos, se omite la comprobación científica de las variables, (Peña, 2007). Adicionalmente, se alteran los niveles de incidencia en los resultados de los procesos, pues la ausencia en la aplicación del diseño de experimentos, reduce la posibilidad de medir la relación entre tratamientos, sistemas, procesos y su impacto real en la producción agrícola.

La aplicación de una herramienta estadística es desarrollada casi exclusivamente en algunos sectores industriales y de servicios, que utilizan los procesos de control de calidad e indicadores de mejora continua, los cuales evolucionan directamente del sector agrícola
(Montgomery, 2011). Sin embargo, en Colombia no se ha tomado como una alternativa de desarrollo, desconociendo la posibilidad de obtener beneficios a través de la utilización de modelos experimentales modernos y contextualizados, con el fin de buscar alternativas de desarrollo con amplias posibilidades de alcanzar niveles de competitividad a nivel mundial.

El objetivo general, es diseñar un modelo experimental mediante bloques aleatorios simples y análisis multivariado, para el mejoramiento de procesos orgánicos en la agroindustria. Para esto, se hará un muestreo aleatorio sistemático, usando la fórmula para universos finitos con tres bloques y cuatro tratamientos con repeticiones, en tres fases de tiempo establecidas, determinando una muestra representativa con un 95\% de confiabilidad; el estudio de las relaciones entre variables, por medio de cuadros de control, pruebas de homogeneidad y diferencias de varianzas (ANAVA) para contrastar hipótesis y el diseño de un modelo utilizando regresión lineal y análisis multivariado, analizando el nivel de representatividad con la realidad y proyectando el crecimiento en diferentes etapas. Lo anterior, permitirá resolver la hipótesis planteada: un modelo experimental con bloques aleatorios simples y análisis multivariado, explica, contrasta y pronostica las variables de estudio que afectan los procesos de crecimiento orgánico.

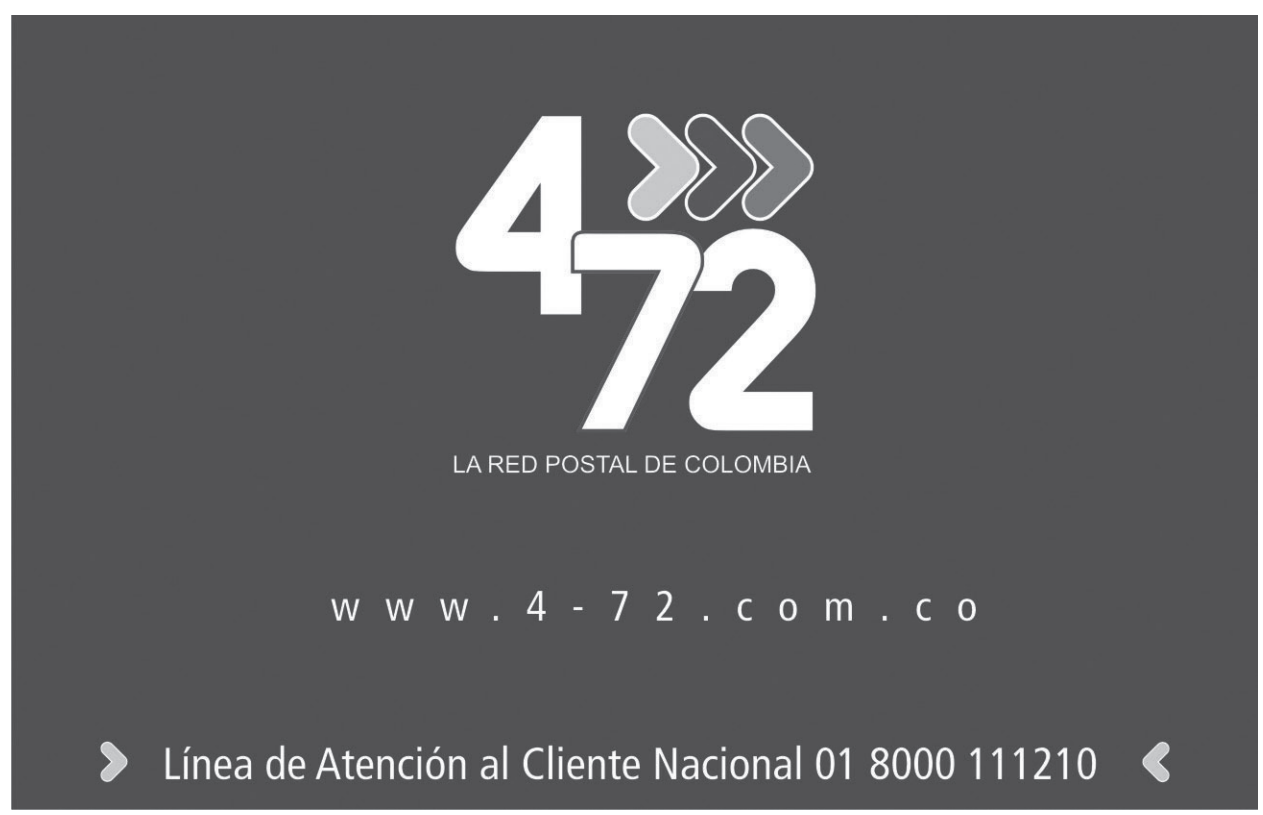




\section{CONCEPTUALIZACIÓN DEL EXPERIMENTO}

L a definición científica de experimento, se refiere a un estudio de investigación en el que se manipulan deliberadamente una o más variables independientes (supuestas causas) para analizar las consecuencias que la manipulación tiene sobre una o más variables dependientes (supuestos efecto), dentro de condiciones controladas por el investigador (Hernández Sampieri, Fernández Collado, \& Baptista Lucio, 2011).

También, se puede decir que un experimento es una investigación planeada para descubrir nuevos hechos o para confirmar o negar los resultados de investigaciones previas (Peña, 2007), base esencial del método científico.

Se debe retomar el concepto de experimentos confirmatorios, que son aquellos en los cuales se trata de comparar el mejor procedimiento encontrado en el experimento exploratorio con un tratamiento establecido o un producto, y determinar si el procedimiento o producto nuevo es mejor que el antiguo (Hinkelman \& Kempphotme, 2008). En el experimento confirmatorio, se quiere encontrar la mejor opción para establecer procesos de control, por ejemplo, sus propiedades estadísticas. También, se conoce que las condiciones pueden cambiar y es importante establecer la media de respuesta y la variabilidad asociada con el proceso.

El diseño experimental es una de las metodologías con más desarrollo y efectividad en el mejoramiento de los procesos. Al estudiar parámetros más reales, en contraste a las técnicas netamente determinísticas o fenomenológicas, se enriquece la toma de decisiones para generar soluciones viables y más funcionales a los procesos. A diferencia del concepto de aplicación actual, los diseños experimentales modernos proceden de la agroindustria, pero en países en vía de desarrollo son más utilizados en el sector manufacturero (multinacionales) y en algunos casos en procesos de certificación de calidad e investigación de mercados. El desconocimiento y la percepción de altos costos han limitado el desarrollo en gran escala de este tipo de metodologías que permiten acabar con la incertidumbre en la toma de decisiones, las falacias administrativas y organizacionales, la pérdida de capital en soluciones no viables, el desconocimiento de variables que afectan procesos, y finalmente la carencia de mejoramiento e innovación científica que afecta la competitividad en el mundo globalizado.

La aplicación de diseño de experimentos en la agroindustria orgánica en Colombia, no está muy desarrollada, debido al incorrecto direccionamiento de inversión en este sector; específicamente en lo que se refiere a procesos no químicos y no transgénicos. Como se mencionó con anterioridad, las políticas están centradas en la estabilización del sector, sin dar paso al desarrollo y lejanas a la innovación.

La falta de conocimiento y de utilización de este tipo de metodologías, como consecuencia de sus altos costos y la restricción en el seguimiento permanente, han hecho que no sean tan frecuentes este tipo de estudios.

No obstante, en Colombia se encuentran algunos ejemplos de estudios desarrollados en esta área, tal como la investigación realizada a través de la Facultad de Ingeniería de la Universidad Nacional de Colombia, titulada: "Procedimientos y condiciones de operación para la deshidratación por ósmosis de pitahaya, mango y espárragos" (Cortés, s.f.). Se realizó una primera fase experimental en la que se fijaron los alcances de la tecnología actual en la deshidratación de pitahaya, y se pusieron a punto el tipo y la metodología de los procedimientos de medida de las variables de respuesta. Finalizada esta primera fase, se diseñó y se construyó un montaje de laboratorio denominado "Prototipo I", en el cual se permitía estudiar simultáneamente las variables de interés: presión, temperatura, tipo de soluto y agitación. Posteriormente, se esbozaron una serie de experimentos cuyos resultados permitieron identificar varias etapas difusivas junto con la influencia de las variables estudiadas en cada una de ellas. 
Otro estudio significativo, corresponde al informe titulado: "Estudio de la influencia de la agitación y concentración de almidón de yuca en la hidrólisis ácida" (Zamora, 2010), el cual expone que tres de los residuos celulósicos agroindustriales más abundantes (paja de trigo, paja de cebada y restos de maíz), fueron sometidos a hidrólisis ácida con ácido sulfúrico, diluido a diferentes concentraciones y diferentes tiempos de reacción, según un diseño experimental $4 \times 3 \times 3$ con tres repeticiones. Los datos obtenidos resultaron ser estadísticamente significativos, e indicaron que es factible obtener cantidades importantes de azúcares a partir de los sustratos estudiados.

Otro estudio de forma similar en el aprovechamiento de residuos orgánicos en el sector agroindustrial, es el mango común (mangifera) en la obtención de azúcares fermentables, en el cual se plantea que el residuo de este (Mangifera indica L.), es un material vegetal que contiene gran cantidad de tejido lignocelulósico, que puede ser aprovechado para la obtención de metabolitos fermentables y productos de la fermentación. En este trabajo se aplicaron tratamientos de hidrolisis al residuo del mango y métodos experimentales para comprobar variables y nivel de interacción entre ellas (Giraldo, Gutiérrez \& Castano, 2007).

Con relación a lo anterior, se puede inferir que: Un diseño de experimento es una prueba o serie de pruebas en las cuales se inducen cambios deliberados en las variables de entrada de un proceso o sistema, de manera que sea posible observar e identificar las causas de los cambios en la respuesta de salida" (Montgomery, 2011). Actualmente, los métodos de diseño experimental tienen una amplia aplicación en muchas disciplinas, por ejemplo en la agricultura y el diseño técnico.

El diseño experimental puede ser considerado como parte del proceso científico y una de las formas en las que se aprende acerca de la manera en que funcionan los sistemas o procesos.

Los modelos explicativos de regresión dinámica que relacionan temporalmente las variables con otras observables, permiten proyectar los resultados de estas interacciones.
La combinación de estas dos metodologías es muy utilizada en la experimentación industrial y permite determinar factores perturbadores de un proceso al establecer cambios por bloque, además de proyectar estos en una simulación determinando consecuencias y disminuyendo costos por factores desconocidos en un proceso (Peña, 2007).

El diseño experimental con modelos multivariados en la agroindustria orgánica, es una alternativa que permite conocer las variables que interactúan en la producción orgánica, como es el caso de las simbiosis de mutualismo.

El carácter mutualista supone también un beneficio para el hongo, que consiste en el suministro, por parte del macro simbionte, de compuestos orgánicos y de un hábitat protegido. Esto significa que la simbiosis tiene un costo energético para la planta en términos de producto fotosintético, costo que normalmente está compensado por las ventajas que trae la asociación (Zubek, Turnau, \& Blaszkowski, 2007).

El sistema micorriza $+P$ insoluble, representa una de las alternativas de mayor futuro para resolver el problema de deficiencia de fósforo que limita el desarrollo agropecuario y silvícola en suelos ácidos del trópico, factor que es el responsable del marginamiento en que hoy permanecen vastas regiones del mundo tropical (García de Pedraza, 1981).

Se ha demostrado que la micorriza favorece, en particular, la absorción de iones poco móviles en el suelo, como H2PO4-, NH4+, $\mathrm{Zn++}$, $\mathrm{Cu}++$, gracias a la elongación del micelio externo más allá de la zona de agotamiento de nutrientes alrededor de la raíz (García, García, \& Correa, 2004). Esto, explica la alta dependencia de la micorriza que manifiestan las plantas cuando crecen sobre suelos pobres en nutrientes. También, se ha observado que reduce la tensión causada por los organismos patógenos de la raíz y aumenta la tolerancia frente a estrés abiótico, salino, hídrico o debido a la presencia de metales pesados (Guerrero, 2005). 


\section{DISEÑO EXPERIMENTAL}

$\mathbf{E}$ diseño experimental corresponde a bloques completos al azar con tres repeticiones por tratamiento. Con la siguiente asignación de micorrizas por tratamiento: T0 $=0 \mathrm{~g}$, (grupo control), $\mathrm{T} 1=20 \mathrm{~g}, \mathrm{~T} 2=40 \mathrm{~g}$ y T3 = $60 \mathrm{~g}$ (Tabla 1); el modelo fue

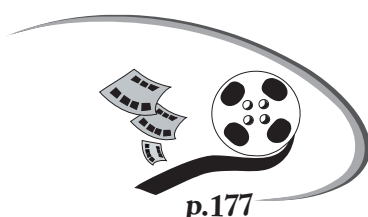
aplicado a plántulas de cafeto (Coffea arabica) con aplicaciones en diferentes cantidades de inóculo de hongos micorrícico arbusculares. La relación de los tratamientos se observa más adelante (Tabla 2).

Tabla 1. Modelo del diseño experimental

\begin{tabular}{|c|c|c|c|c|c|c|c|c|c|c|c|}
\multicolumn{1}{c}{ R1 } & R2 & \multicolumn{1}{c|}{ R3 } \\
\hline T1 & T0 & T3 & T2 & T0 & T2 & T1 & T3 & T3 & T1 & T0 & T2 \\
\hline
\end{tabular}

Fuente. Elaboración propia (s.f.).

Tabla 2. Relación de los tratamientos

\begin{tabular}{|c|c|c|}
\hline № Tratamiento & Cantidad (gr.) & $\begin{array}{l}N^{\circ} \text { de plántulas } \\
\text { por repetición }\end{array}$ \\
\hline 0 & 0 & 50 \\
\hline 1 & 20 & 50 \\
\hline 2 & 40 & 50 \\
\hline 3 & 60 & 50 \\
\hline
\end{tabular}

Fuente. Elaboración propia (s.f.).

\subsection{Muestreo}

El número total de plántulas en cada ensayo fue de 600. Para determinar el número de muestras que sería representativo o que se tenía que muestrear, en cada monitoreo se utilizó la fórmula para muestra de frecuencia normal (Peña, 2007), ya que se parte del principio de normalidad del fenómeno. Este número se determinó por medio de la fórmula:

$$
\mathrm{n}=\mathrm{Z2}(\mathrm{PQ}) \mathrm{N} / \mathrm{E} 2(\mathrm{~N}-1)+\mathrm{Z} 2(\mathrm{PQ})
$$

En donde:

n: número necesario a muestrear

Z: valor de la tabla de factor normal
P: posibilidad de éxito
Q: posibilidad de fracaso
$\mathrm{N}$ : universo
E: error

Con un grado de confiabilidad del $95 \%$ y un margen de error de $5 \%$, se halló que el $n=180$. Por lo tanto, en cada monitoreo se registraron mediciones para cinco muestras, en tres meses, dando un total de 60 muestras en todo el proceso por cada tratamiento.

Así mismo, se partió de las hipótesis:

- Hipótesis nula $\left(\mathrm{H}_{0}\right)$ : no existen diferencias entre las medias de ningún tratamiento.

- Hipótesis alternativa $\left(\mathrm{H}_{1}\right)$ : si existen diferencias significativas entre una o más medias de los tratamientos.

\subsection{Desarrollo del experimento}

Se realizó un análisis de varianza (ANAVA) de una vía para el diseño experimental por cada fecha de muestreo, con el cual se pudo establecer si existían o no diferencias estadísticamente significativas para cada tratamiento en las variables dependientes: longitud del sistema radical, altura de la planta, diámetro del tallo y biomasa. Adicionalmente a los resultados que presentaron diferencias estadísticamente significativas, se les realizó la prueba Fischer - LSD de diferencia de medias, la cual determinó el valor mínimo necesario para considerar diferencias en los dos tratamientos. Posteriormente, se realizaron las pruebas de comparaciones múltiples de Tukey y el Test de Duncan por medio del programa estadístico Statistics Programer Service System (SPSS ${ }^{\circledR}$ ).

Se realiza la prueba de normalidad $K-S$, para determinar el tipo de frecuencia en que se comportan las variables (Tabla 3). 
Tabla 3. Prueba de normalidad

\begin{tabular}{|c|c|c|c|c|c|c|c|c|}
\hline \multicolumn{9}{|c|}{ Prueba de Kolmogorov-Smirnov para una muestra } \\
\hline & & Tratamiento & Repetición & Total & Aerea & Raiz & Diametro & Biomasa \\
\hline $\mathrm{N}$ & & 60 & 60 & 60 & 60 & 60 & 60 & 60 \\
\hline \multirow{2}{*}{$\begin{array}{l}\text { Parámetros } \\
\text { normales } a, b\end{array}$} & Media & 1,5000 & 2,0000 & 18,4917 & 6,9783 & 11,5133 & ,2445 & ,9882 \\
\hline & $\begin{array}{l}\text { Desviación } \\
\text { típica }\end{array}$ & 1,12747 & ,82339 & 2,17702 & ,76271 & 1,99240 & 02127 & ,25679 \\
\hline \multirow{3}{*}{$\begin{array}{l}\text { Diferencias más } \\
\text { extremas }\end{array}$} & Absoluta & 171 &, 221 & 068 &, 120 &, 110 &, 184 & 060 \\
\hline & Positiva &, 171 & 221 & 053 &, 120 & 076 &, 184 &, 060 \\
\hline & Negativa &,- 171 &,- 221 &,- 068 &,- 059 &,- 110 &,- 101 &,- 059 \\
\hline $\begin{array}{l}\text { Z de Kolmogorov- } \\
\text { Smirnov }\end{array}$ & & 1,327 & 1,712 &, 528 & 929 & 854 & 1,424 & 466 \\
\hline \multicolumn{2}{|l|}{ Sig. asintót. (bilateral) } & 059 & ,006 & 943 &, 354 & 459 & 035 & 982 \\
\hline \multicolumn{9}{|c|}{ a. La distribución de contraste es la Normal. } \\
\hline \multicolumn{9}{|c|}{ b. Se han calculado a partir de los datos. } \\
\hline
\end{tabular}

Fuente. Elaboración propia, (s.f.).

A partir de la prueba Kolmogorov-smirnov se estblece que el comportamiento de las variables es normal, permitiendo aplicar pruebas a las hipótesis, basadas en el supuesto de normalidad.
Así mismo, se aplicó una prueba de colinealidad para determinar la correlación entre variables, la cual permite delimitar la relación entre las variables independientes y las variables de estudio. Para este caso la prueba de colinealidad permitió descartar variables sustituibles o que ejercen la misma función afectando el análisis explicativo del experimento (Tabla 4). 
Tabla 4. Prueba de colinealidad

\begin{tabular}{|c|c|c|c|c|c|c|c|c|c|}
\hline & Tratamiento & & Raíz & Repetición & Total & Diámetro & Área & Biomasa & Área Foliar \\
\hline \multirow{3}{*}{ Tratamiento } & $\begin{array}{l}\text { Correlación } \\
\text { de Pearson }\end{array}$ & 1 & ,029 & ,000 & 107 & , 102 & 228 &,- 093 &.$a$ \\
\hline & Sig. (bilateral) & & ,823 & 1,000 & ,417 & ,436 & ,080 &, 478 & \\
\hline & $\mathrm{N}$ & 60 & 60 & 60 & 60 & 60 & 60 & 60 & 0 \\
\hline \multirow{3}{*}{ Raíz } & $\begin{array}{l}\text { Correlación } \\
\text { de Pearson }\end{array}$ & ,029 & 1 &, $359 * *$ &, $937 * *$ &,- 007 & ,062 & ,210 & . a \\
\hline & Sig. (bilateral) &, 823 & &, 005 &, 000 &, 955 & ,639 & , 107 & \\
\hline & $\mathrm{N}$ & 60 & 60 & 60 & 60 & 60 & 60 & 60 & 0 \\
\hline \multirow{3}{*}{ Repetición } & $\begin{array}{l}\text { Correlación } \\
\text { de Pearson }\end{array}$ & ,000 &, $359 * *$ & 1 &, $381 * *$ &,- 097 & , 151 & ,253 & a \\
\hline & Sig. (bilateral) & 1,000 & ,005 & &, 003 &, 462 & ,249 &, 051 & . \\
\hline & $\mathrm{N}$ & 60 & 60 & 60 & 60 & 60 & 60 & 60 & 0 \\
\hline \multirow{3}{*}{ Total } & $\begin{array}{l}\text { Correlación } \\
\text { de Pearson }\end{array}$ & , 107 & $937 * *$ &, $381 * *$ & 1 & ,059 &, $407 * *$ &, $371 * *$ & a \\
\hline & Sig. (bilateral) & ,417 &, 000 &, 003 & & ,652 &, 001 &, 003 & . \\
\hline & $\mathrm{N}$ & 60 & 60 & 60 & 60 & 60 & 60 & 60 & 0 \\
\hline \multirow{3}{*}{ Diámetro } & $\begin{array}{l}\text { Correlación } \\
\text { de Pearson }\end{array}$ & , 102 &,- 007 &,- 097 & ,059 & 1 & , 189 & ,291* & . $a$ \\
\hline & Sig. (bilateral) & ,436 & ,955 &, 462 & ,652 & &, 148 &, 024 & \\
\hline & $\mathrm{N}$ & 60 & 60 & 60 & 60 & 60 & 60 & 60 & 0 \\
\hline \multirow{3}{*}{ Área } & $\begin{array}{l}\text { Correlación } \\
\text { de Pearson }\end{array}$ & 228 & ,062 & , 151 & $407^{\star \star}$ & 189 & 1 &, $511 * \star$ & a \\
\hline & Sig. (bilateral) &, 080 & ,639 & ,249 &, 001 & 148 & &, 000 & . \\
\hline & $\mathrm{N}$ & 60 & 60 & 60 & 60 & 60 & 60 & 60 & 0 \\
\hline \multirow{3}{*}{ Biomasa } & $\begin{array}{l}\text { Correlación } \\
\text { de Pearson }\end{array}$ &,- 093 & ,210 & ,253 &, $371 * *$ & ,291* &, $511 * \star$ & 1 & . $a$ \\
\hline & Sig. (bilateral) & ,478 & , 107 & ,051 &, 003 &, 024 &, 000 & & . \\
\hline & $\mathrm{N}$ & 60 & 60 & 60 & 60 & 60 & 60 & 60 & 0 \\
\hline \multirow{3}{*}{ Área Foliar } & $\begin{array}{l}\text { Correlación } \\
\text { de Pearson }\end{array}$ & .a & . $a$ & .a & . a & .a & .a &.$a$ & . $a$ \\
\hline & Sig. (bilateral) & . & . & & . & . & . & . & \\
\hline & $\mathrm{N}$ & 0 & 0 & 0 & 0 & 0 & 0 & 0 & 0 \\
\hline \multicolumn{10}{|c|}{ * * . La correlación es significativa al nivel 0,01 (bilateral). } \\
\hline & & *. La & correlacic & n es significar & te al nivel & ),05 (bilater & & & \\
\hline
\end{tabular}

\section{Fuente. Elaboración propia (s.f.).}

La correlación más alta con un 0.9 está entre variables de salida tamaño de raíz y altura, esto significa que el tratamiento afecta en un 0.9 a éstas dos variables de salida.
Lo anterior permite demostrar que se puede explicar la relación entre las variables tratamiento y tiempo frente a las variables de salida, debido a que no se presenta colinealidad se puede generar un modelo que explique la interacción de las mismas y su proyección en tiempo. 
A continuación, se muestra la representación gráfica de la prueba de ANAVA para la variable longitud del sistema radical como resultado de la aplicación del modelo (Figura 1).

Figura 1. Prueba de ANAVA para la variable longitud del sistema radical
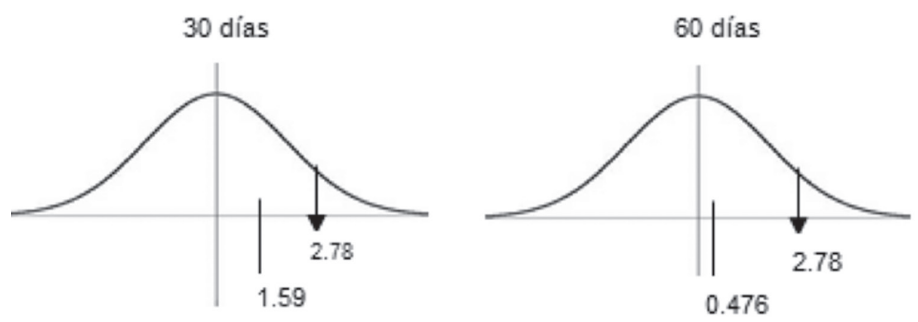

Fuente. Elaboración propia (s.f.).

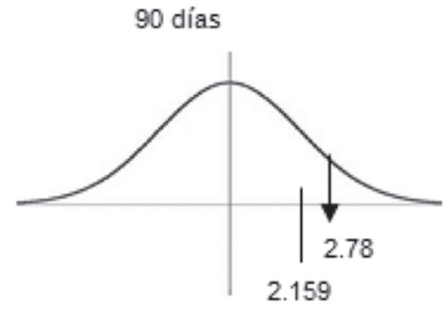

La variable tiempo, como se puede apreciar, muestra los resultados de la prueba de varianza e indica que a los 30 días no se determina una diferencia significativa en las medias de los tratamientos, por lo tanto se acepta la hipótesis nula (no existe diferencia de medias); sin embargo, a los 60 días y a los 90 días, se evidencian diferencias estadísticas representativas entre los tratamientos, aceptando la hipótesis alterna de diferencia de las medias (Figura 2).

\section{Figura 2. Diferencias de medias por tratamiento}

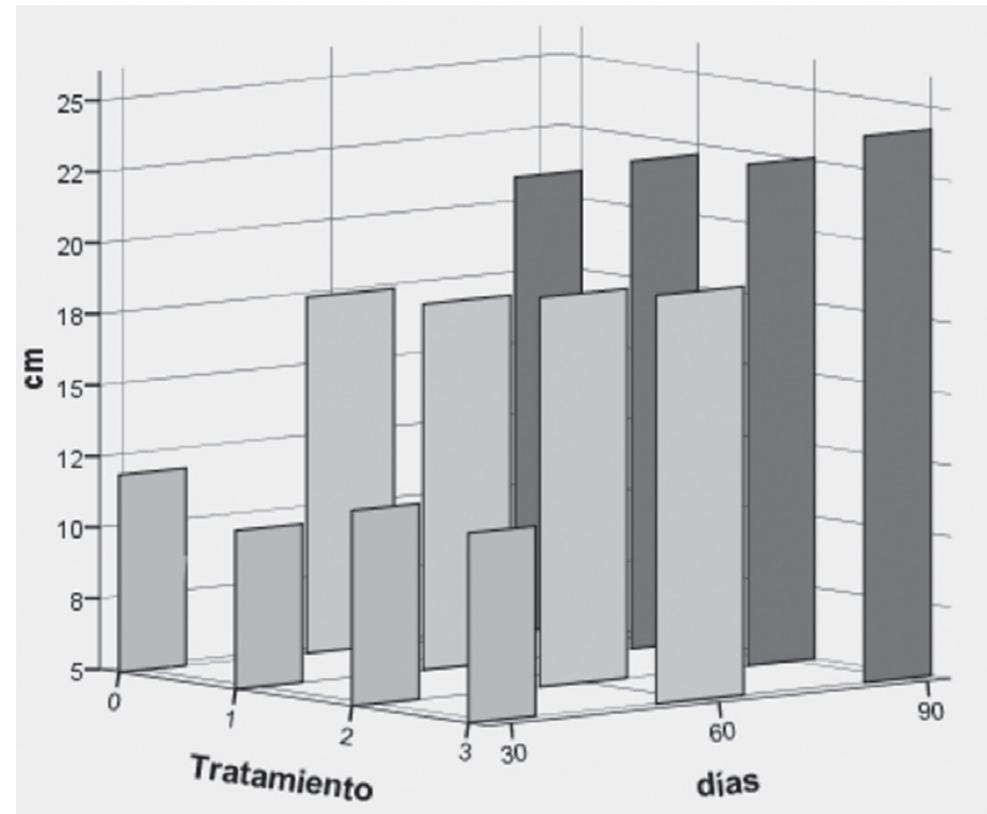

Fuente. Elaboración propia (s.f.). 
La dinámica de crecimiento en centímetros, que tuvieron las plántulas con los tratamientos en los diferentes días, permite identificar que para el día 30, el grupo control fue el que presentó un mayor aumento con un valor de $11.94 \mathrm{~cm}$ en promedio.

En contraste, el día 60 fue el tratamiento tres el que registro un mejor resultado, con $19.36 \mathrm{~cm}$ de crecimiento en promedio y el grupo control fue el menor con $17.51 \mathrm{~cm}$, sin embargo, para esta fecha no hay evidencia suficiente para rechazar la hipótesis nula.
Así mismo, se señalan los resultados obtenidos el día 90, en donde nuevamente el tratamiento tres con 60gr de inoculo proporcionó mejores resultados con $24.21 \mathrm{~cm}$ de crecimiento en longitud de la raíz.

A continuación, por medio de la prueba de diferencia de medias LSD de Fisher, se determina la diferencia entre las medias de los diferentes tratamientos, lo cual permite demostrar que el tratamiento tres, mejora los rendimientos en 90 días frente a las otras opciones de tratamiento (Tabla 5).

Tabla 5. Diferencia de medias Fischer - LSD, para longitud del sistema radical 90 días

\begin{tabular}{|c|c|c|c|c|c|c|}
\hline \multirow{2}{*}{ (I) Tratamiento } & \multirow{2}{*}{ (J) Tratamiento } & \multirow{2}{*}{$\begin{array}{l}\text { Diferencia de } \\
\text { medias (I-J) }\end{array}$} & \multirow{2}{*}{$\begin{array}{c}\text { Error } \\
\text { estadístico }\end{array}$} & \multirow{2}{*}{ Sig. } & \multicolumn{2}{|c|}{ 95\% Intervalo de confianza } \\
\hline & & & & & Límite inferior & Límite superior \\
\hline \multirow{3}{*}{0} & 1 & .67333 & 1.33407 & .616 & -1.9991 & 3.3458 \\
\hline & 2 & -.56000 & 1.33407 & .676 & -3.2325 & 2.1125 \\
\hline & 3 & $-3.52000^{*}$ & 1.33407 & .011 & -6.1925 & -.8475 \\
\hline \multirow{3}{*}{1} & 0 & -.67333 & 1.33407 & .616 & -3.3458 & 1.9991 \\
\hline & 2 & -1.23333 & 1.33407 & .359 & -3.9058 & 1.4391 \\
\hline & 3 & $-4.19333^{\star}$ & 1.33407 & .003 & -6.8658 & -1.5209 \\
\hline \multirow{3}{*}{2} & 0 & .56000 & 1.33407 & .676 & -2.1125 & 3.2325 \\
\hline & 1 & 1.23333 & 1.33407 & .359 & -1.4391 & 3.9058 \\
\hline & 3 & $-2.96000^{*}$ & 1.33407 & .031 & -5.6325 & -.2875 \\
\hline \multirow{3}{*}{3} & 0 & $3.52000^{*}$ & 1.33407 & .011 & .8475 & 6.1925 \\
\hline & 1 & $4.19333^{*}$ & 1.33407 & .003 & 1.5209 & 6.8658 \\
\hline & 2 & $2.96000^{*}$ & 1.33407 & .031 & .2875 & 5.6325 \\
\hline
\end{tabular}

Fuente. Elaboración propia (s.f.).

Por su parte, la prueba de comparaciones múltiples de Tukey arroja dos subconjuntos de homogeneidad de medias. En el primer subconjunto se encuentran el testigo y los tratamientos uno y dos, y en el segundo subconjunto los tratamientos uno, dos y tres. El test de Duncan proporciona igualmente dos subconjuntos en donde compara las medias de los tratamientos, pero es mucho más excluyente y muestra en el segundo subconjunto solamente a el tratamiento tres, hecho que valida la confirmación de la hipótesis alternativa y lo señala como el de mejores resultados.

Se aplican cuadros de control en el crecimiento de la planta dando los siguientes resultados que muestran los comparativos del grupo control frente al tratamiento tres, en la variable altura de la planta (Figura 3). 
Figura 3. Cuadros de control comparativo por altura de la planta. Tratamiento 0 (grupo control) vs tratamiento tres
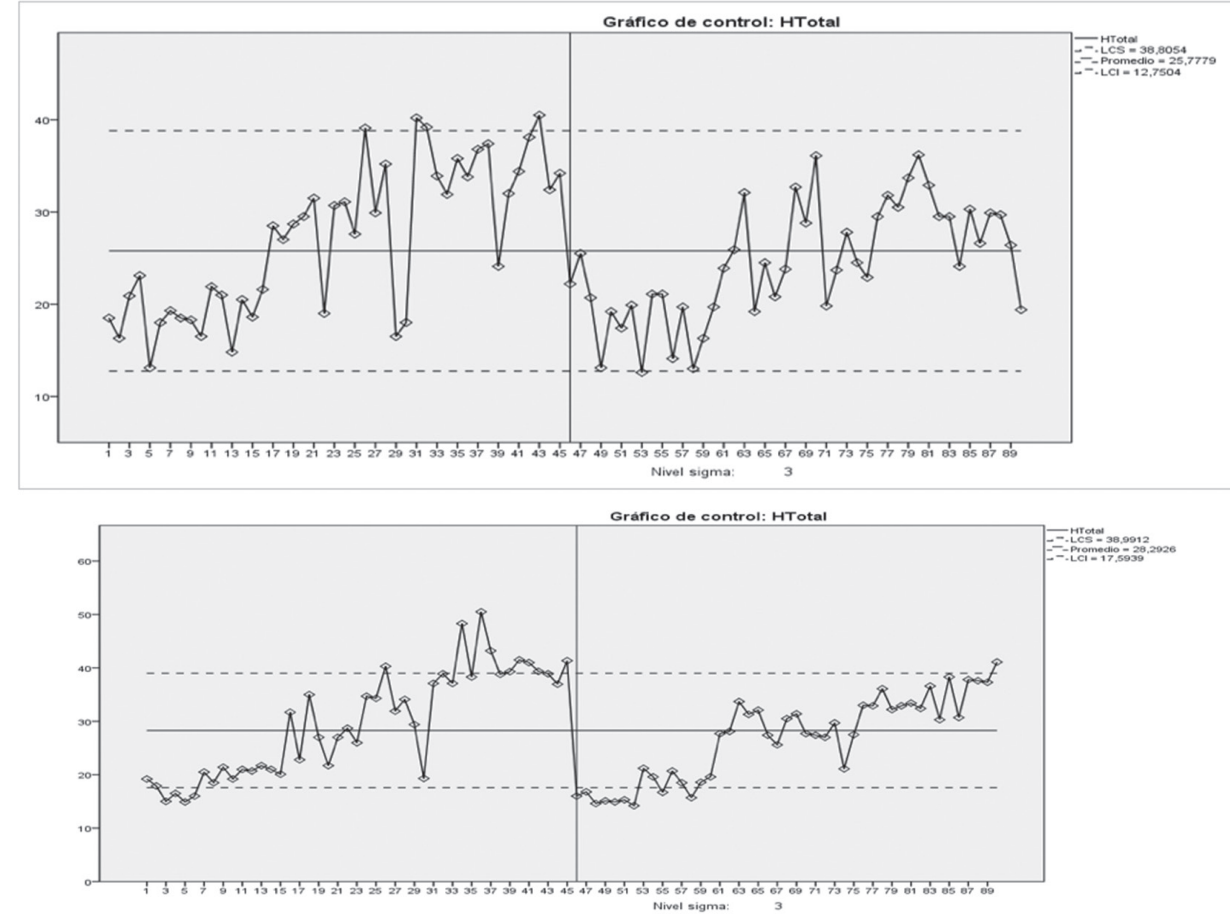

\begin{tabular}{|c|l|l|c|c|c|c|}
\hline \multicolumn{2}{|c|}{} & \multicolumn{1}{|c|}{$\begin{array}{c}\text { Suma de } \\
\text { cuadrados }\end{array}$} & gl & $\begin{array}{c}\text { Media } \\
\text { cuadrática }\end{array}$ & F & Sig. \\
\hline \multirow{3}{*}{1} & Regresión & 16871,603 & 3 & 5623,868 & 271,587 &, 000 a \\
\cline { 2 - 7 } & Residual & 7371,854 & 356 & 20,707 & & \\
\cline { 2 - 7 } & Total & 24243,457 & 359 & & & \\
\hline
\end{tabular}

Fuente. Elaboración propia (s.f.).

Se determina una diferencia significativa en la altura de la plántula; en el tratamiento 0 , un valor de $25.77 \mathrm{~cm}$, mientras en el tratamiento tres un valor medio de 28.29 con una diferencia de crecimiento de $2.52 \mathrm{~cm}$.

Los cuadros de control, como en este ejemplo permiten llevar un registro permanente del comportamiento de las variables dependientes.

\section{- Modelo multivariado}

A continuación se determinan las variables independientes para la formulación de un modelo multivariado que representa este proceso:
$Y=B 0+B 1 * B 1+B 2+x 2+B 3 * x 3+$ eij

Donde:

$\mathbf{B O}=$ constaste del sistema

$\mathbf{B 1}=$ es el valor de afectación de la variable 1

$\mathbf{B 2}=$ es el valor de afectación de la variable 2

B3= es el valor de afectación de la variable 3

$\mathbf{e}=$ error del modelo

Se generó un análisis hacia atrás, eliminando las variables de menor nivel de incidencia y mayor significancia del sistema para el Modelo, con el fin de ajustar y aumentar su nivel $\mathrm{R}^{2}$, que indica la incidencia sobre las variables dependientes (Tabla 7). 
Tabla 6. Modelo-variables eliminadas

\begin{tabular}{|c|l|l|l|l|}
\hline \multicolumn{5}{|c|}{ Variables introducidas/eliminadas $^{\mathbf{b}}$} \\
\hline Modelo & & \multicolumn{1}{|c|}{ Variables introducidas } & $\begin{array}{c}\text { Variables } \\
\text { eliminadas }\end{array}$ & Método \\
\hline dimension & 1 & tiempo de observacion, repeticion, tratamientoa &. & Introducir \\
\hline
\end{tabular}

Fuente. Elaboración propia (s.f.).

Tabla 7. Resumen del modelo

\begin{tabular}{|l|l|l|l|l|l|}
\hline \multicolumn{1}{|c|}{ Modelo } & \multicolumn{1}{|c|}{ RESUMEN DEL MODELO } \\
\hline dimensión & 1 & R cuadrado & $\begin{array}{c}\text { R cuadrado } \\
\text { corregida }\end{array}$ & $\begin{array}{c}\text { Error típ. de la } \\
\text { estimación }\end{array}$ \\
\hline a. Variables predictoras: (Constante), tiempo de observación, Repetición, Tratamiento & 4,55054 \\
\hline
\end{tabular}

Fuente. Elaboración propia (s.f.).

El Coeficiente de Pearson $\left(R^{2}\right)$ menciona que el $70 \%$ de las variaciones del crecimiento son explicadas por el Modelo.
El nivel de significancia de la prueba ANOVA permite afirmar que el modelo representa la variable dependiente. En este caso, altura total de la planta.

Tabla 8. Coeficientes del Modelo

\begin{tabular}{|c|c|c|c|c|c|c|}
\hline \multicolumn{7}{|c|}{ Coeficientes $^{\mathrm{a}}$} \\
\hline Modelo & & \multicolumn{2}{|c|}{$\begin{array}{l}\text { Coeficientes no } \\
\text { estandarizados }\end{array}$} & $\begin{array}{c}\text { Coeficientes } \\
\text { tipificados }\end{array}$ & \multirow[t]{2}{*}{$\mathrm{t}$} & \multirow[t]{2}{*}{ Sig. } \\
\hline \multirow{5}{*}{1} & & B & Error típ. & Beta & & \\
\hline & (Constante) & 8,409 & ,923 & & 9,114 &, 000 \\
\hline & Tratamiento & 882 & ,215 & ,120 & 4,112 &, 000 \\
\hline & Repeticion & ,265 & ,294 &, 026 & ,902 & ,368 \\
\hline & $\begin{array}{l}\text { tiempo de } \\
\text { observacion }\end{array}$ & 8,293 & ,294 &, 825 & 28,232 & ,000 \\
\hline
\end{tabular}

Fuente. Elaboración propia (s.f.).

La variable con mayor nivel de significancia, es repetición, por lo tanto es eliminada del Modelo.

Quedando la ecuación $Y=8.409+0.882 * T x+8.293 * T x+0.477$

Con un $\mathrm{R}^{2}=70 \%$ 
Figura 4. Modelo proyectado y ajustado vs. la realidad
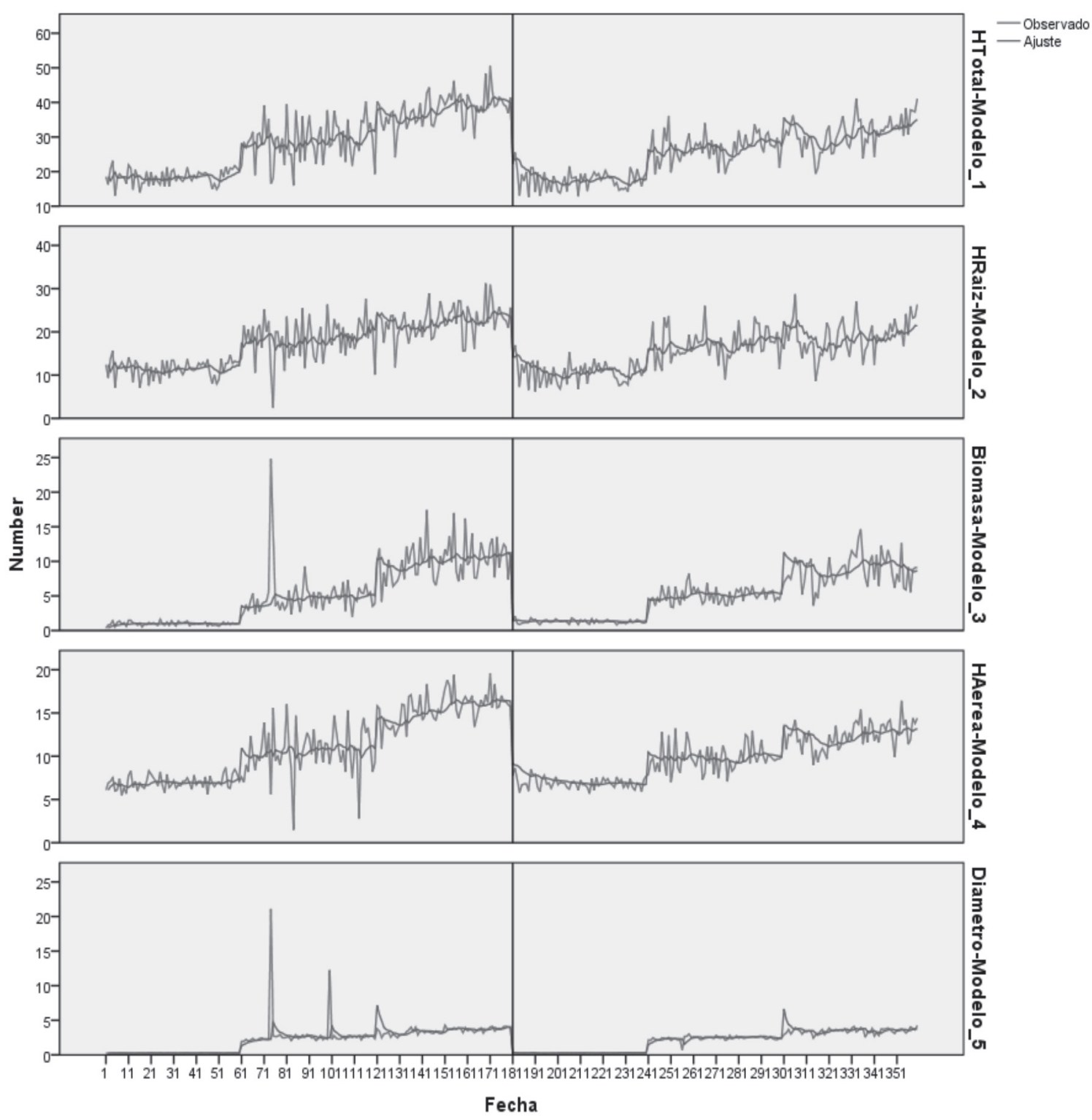

Fuente. Elaboración propia (s.f.).

El Modelo, comparado con la realidad, representa el valor medio de las variables dependientes y cumple la función de proyección en diferentes estadios de tiempo y crecimiento en las plántulas de café. 


\section{DISCUSIÓN}

J as prácticas de la agroindustria se mantienen en estadios del empirismo, con mediciones en indicadores asociados a factores nominales como las cantidades producidas, sin embargo, los mercados mundiales se que concentran en la sostenibilidad, la producción limpia y la calidad de los productos; en la industria, desde la formulación de las políticas, así como en el sector productivo las mediciones estándares se limitan a la producción y la extensión de tierra. Lo anterior tiene como consecuencia un distanciamiento entre las actividades de desarrollo científico y la agricultura entendida como una práctica social, artesanal y económica, que representa ingresos para las familias de las áreas rurales pero con bajos niveles de sofisticación y sin mejoramiento de los procesos.

Al considerar la agricultura como una industria, se deberían incluir el reconocimiento de nichos de mercado en procesos no químicos y no transgénicos, los cuales están por explorar y como se comprobó en el caso de experimentación con bloques aleatorios y análisis multivariado, permitió constatar que el uso de micorrizas en tratamientos por grupos en cantidades específicas, mejora el crecimiento de la altura de la planta y por lo tanto, hace eficiente el proceso productivo y permite la optimización de los recursos, los cuales, por principio económico, son escasos.

Una de las ventajas que ofrece el modelo de experimentación, es la integración entre el tratamiento, el sistema, el proceso y su impacto en la producción, lo que se refleja en la calidad, entrecruzando las variables nominales con las reales; es decir, la eficiencia económica no sólo dependerá de la cantidad producida, lo cual limita a economías de escala, sino a la calidad y optimización de los recursos, es decir, economías de alcance, al aprovechar un insumo natural en la producción de otro bien sostenible minimizando el costo y siendo amigable con el medio ambiente.
Aunque la historia ha demostrado que a mayor nivel de desarrollo industrial del país, hay menor dedicación al sector agrícola, se reconoce la importancia del sector en la política agroalimentaria como un factor de desarrollo social. Por lo tanto, es un tema prioritario en la agenda mundial y exige un papel renovado no sólo para el desarrollo de demanda agregada, es decir, subsidiario de otros sectores, sino hacia la generación de cadenas de valor integradas e innovación tecnológica; desde el sentido amplio, se explica cómo las nuevas formas de producción, superando las limitantes ambientales de las industrias tradicionales químicas y trasgénicas, como se ha discutido en las políticas de desarrollo de la agricultura en Colombia, (Perfetti, Balcázar, Hernández, \& Leibovich, 2013).

La composición de la agroindustria presenta algunas limitantes para la aplicación de la actividad científica, la cual puede ser superada por el empoderamiento desde la institucionalidad que busca la promoción, el fomento y el mejoramiento de los procesos agrícolas. En la caracterización del sector, es evidente la existencia de pequeños productores, la desutilización del potencial en suelos en un 24,1 según el Instituto Geográfico Agustín Codazzi (IGAC, 2012), bajos niveles de diversificación y concentración como medida de producción en la cantidad sin estándares de calidad. Por lo tanto, se presenta como aporte el uso de sistemas de experimentación, haciendo uso de bloques aleatorios simples y análisis multivariado, el cual puede ser una alternativa para la medición de las variables asociadas al proceso para el caso de un producto como el café, haciendo uso de insumos orgánicos y bajo los principios de la sostenibilidad.

Dentro de los factores que se han mencionado como impulsores del sector agropecuario cabe mencionar las condiciones climáticas, el potencial en suelos, los factores comerciales, la redistribución de la 
tierra y el reconocimiento como factor de desarrollo social para la reducción de la pobreza y la inclusión; sin embargo, están por explorar otros factores de tipo cuantitativo que afectan la cadena de valor y el rendimiento de las cosechas, como se demostró en el caso particular presentado de experimentación, los cuales son los insumos para la generación de productos orgánicos, que pueden estar asociados a prácticas ancentrales de tipo empírico y son objeto de actividad científica al demostrar la mejora en el rendimiento.

\section{CONCLUSIONES}

$\mathbf{E}$ I experimento determina que existe evidencia estadística para afirmar que el modelo experimental con bloques aleatorios simples y análisis multivariado, es una herramienta fundamental para inferir, explicar, medir y proyectar las variables que afectan el desarrollo de los procesos estudiados en este; determinando cual es el mejor tratamiento y su incidencia en el crecimiento de las plantas de café; dando respuesta a la pregunta de investigación.

El diseño experimental, es una metodología explicativa que permite generar escenarios proyectados que al compararlo con la realidad, logra explicar en un $76 \%$ el resultado de cada tratamiento y las variables de mayor incidencia en cada proceso. Es así como da una base fundamental para la optimización.

Es una herramienta fundamental para la toma de decisiones, ya que al permitir entender la sinergia de cada proceso, da parámetros para medir, cuantificar y direccionar las variables más relevantes, en función del cumplimiento de objetivos organizacionales.
Se puede cambiar el concepto de costo beneficio de esta técnica, ya que se ahorraron más del $49 \%$ del capital con el experimento, puesto que al invertir en medición y control se bajaron costos, al disminuir la perdida de insumos y muertes de plantas. De esta manera

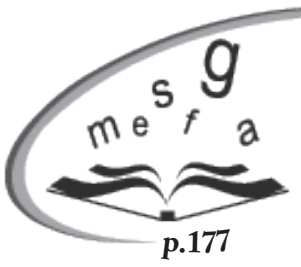
se optimizaron resultados por medio de una técnica orgánica

La ingeniería industrial, contribuye al sector agroindustrial, determinando alternativas de solución a problemas reales generando un mejor direccionamiento frente a las alternativas en ese tipo de cultivos, lo cual puede ser aplicable a diferentes variables como: insumos, suelos, capacitación, proyección de crecimiento, optimización de recursos, entre otros.

La integración exitosa de una buena práctica del diseño experimental en ingeniería y las ciencias es un factor clave en la competitividad industrial futura Métodos experimentales" (Mongomery, 2011). 


\section{REFERENCIAS BIBLIOGRÁFICAS}

Cortés G., J. H. (s.f.). Procedimientos y Condiciones de Operación para la Deshidratación por Ósmosis de Pitahaya, Mango y Espárragos. Bogotá D.C.: Universidad Nacional de Colombia.

DPN, D. d. (13 de Noviembre de 2014). Información agricola. Recuperado el 2 de Febrero de 2015, de https://www. dnp.gov.co/programas/agricultura/estadisticas-del-sector-agropecuario/Paginas/informacion-agricola.aspx

DPN, D. d. (26 de Enero de 2015). Misión para la Transformación del Campo Colombiano. Recuperado el 2 de Febrero de 2015, de https://www.dnp.gov.co/programas/agricultura/Paginas/mision-para-la-transformaciondel-campo-colombiano.aspx

Echavarria, A. (1998). La formación Profesional Especifica. Madrid: Santillana.

Fonseca Santillana, E. B., Oviedo, A. M., \& Vargas, I. J. (2006). HIDRÓLISIS ACIDA DE SUSTRATOSRESIDUALESAGROINDUSTRIALES COLOMBIANOS. Bogotá D.C.: Fundación Universitaria Manuela Beltrán.

García de Pedraza, L. (1981). El clima agrícola de un lugar. . Bogotá: Hojas Divulgadoras-Ministerio de Agricultura.

García, J., García, D., \& Correa, M. (2004). Incidencia de las micorrizas arbusculares y vesículo arbusculares como estrategia adaptativa de especies de páramo y selva altoandina, cordillera oriental de Colombia. Colombia Forestal, 8(17), 43-59.

Giraldo, L. M., M, C. L., Gutiérrez J, B., \& Castano. (2007). Aprovechamiento del residuo agroindustrial del mango común (Mangifera indica L.) en la obtención de azúcares fermentables. Ingeniería y ciencia.

Gómez Tovar, L., \& Cruz, M. (2004). La agricultura orgánica en México: Un ejemplo de incorporación y resistencia a la globalización. Oaxaca, México.

Guerrero, M. G. (2005). Estudio de los mecanismos implicados en la homeostasis de metales pesados en el hongo formador de micorrizas arbusculares" Glomus intraradices": tesis doctoral. . Granada: Editorial Universidad de Granada.

Hernández Sampieri, R., Fernández Collado, C., \& Baptista Lucio, P. (2011). Metodología de investigación. México: Mc Graw Hill.

Hinkelman, K., \& Kempphotme, O. (2008). Desing and analysis of experiments. Ed. Jhonn Wiley Pag 1-52. Vol 1.

Jaramillo, C. F. (1990-2000). Crisis y transformación de la agricultura colombiana. Bogotá: Ed. Banco de la Republica.

Jaramillo, C. F. (2000). Crisis y Transformación de la Agricultura Colombiana.

Mejía Giraldo, L. F., Martinez Correa, H. A., Betancourt Gutiérrez, J. E., \& Castrillón Castaño, C. E. (2007). aprovechamiento del residuo agroindustrial del mango común (mangifera indica l.) en la obtención de azúcares fermentables. Medellín: Universidad EAFIT Facultad de Ingeniería.

Montgomery, D. (2011). Diseños y análisis de experimentos. México: Ed 5 a Limusa S.A.

Peña, D. (2007). Estadística: Modelos y métodos. S.A. Madrid: Ed 2ª Alianza Editorial .

Zamora, L. L. (2010). Estudio de la influencia de la agitación y concentración de almidón de yuca en la hidrólisis ácida. México.

Zubek, S., Turnau, K., \& Blaszkowski, J. (2007). Arbuscular mycorrhiza of endemic and endangered plants from the Tatra Mts. Acta Societatis Botanicorum Poloniae. 